\title{
Cardiovascular effects of paced respiration and selective attention ${ }^{1}$
}

HIRAII L. FIT/GERALD and STIPHLN W. PORGitS, Michigan State University, liast l.ansing, Mich. 48923

The effects of paced respiration (PR) and attentive observation (ATT) on heart rate $(H R)$ and finger-pluse amplitude (FPA) were investigated in adult female $S$ s. Although HR response's to trial onset were task dependent, accelerating for (irmip) $P R$ and decelerating for Group ATT. temperally conditioned anticipatory $H R$ deceleration was obtained across tasks. Across trials, HR decelerated and FPA dilated, indicating autonomic habituation to the experimental conditions. Methodological implications for paced respiration research and FPA analysis were discelssed.

Paced respiration is a technique used to investigate the relationship between respiratory and cardiovascular responses. During paced respiration, Ss pace their breathing rate 10 that of an exterral stimulus that is typically presented in the visual or auditory modality. Using this technique, Brener \& Hothersall (1966) showed that high heart rates (HR) were associated with quick erratic breathing, while low HRs were associated with normal breathing. Hnatiow \& Lang (1965) observed a "weak relationship" between respiratory cycle time and HR variability. Porges \& Raskin (1969) found that, although the direction of the HR response appears to be independent of respiratory responses, HR variability decreases concomitantly with increases in respiratory frequency and decreases in respiratory amplitude.

Previous research has suggested that the direction of the $H R$ response is task dependent: HR acceleration accompanying cognitive tasks and HR deceleration accompanying perceptual tasks (Lacey, 1959; Porges \& Raskin, 1969). Most studies that have used the paced-respiration technique have not controlled for the effects of selective attention to the pacing stimulus. Thus, it is not clear whether the effects of paced respiration are due to $\mathrm{S}$ s attending to the pacing cue or to the requirements of the pacing task, i.e., are these effects task dependent? The first purpose of the present study was to investigate the effects of paced respiration and selective attention on two cardiovascular responses that are strongly influcnced by psychological processes: HR and finger-pulse amplitude (FPA).
The use of a tixed intertrial interval (ITI) represents the Pavlovian procedure for demonstrating temporal conditioning, the fixed ITI serving as a temporal conditional stimulus (CS). Fitzgerald, Lintz, Brackbill, \& Adams (1967) reported successful temporal conditioning of an autonomic response (pupillary reflex) in human infants, while Lipsitt \& Ambrose (1967) obtained temporal conditioning of an autonomic (HR) and a somatic response (body activity). Lockhart (1966) temporally conditioned GSR with adult Ss, and Gantt (1946) reported successful temporal conditioning of adult HR. It would appear that both somatically mediated and autonomically mediated responses are subject to temporal conditioning. But the picture is not that clear. For example, Brackbill, Lintz, \& Fitzgeraid (1968) failed to obtain temporally conditioned eyeblinking - in infants, and Abrahamson, Brackbill, Carpenter, \& Fitzgerald (in press) were equally unsuccessful in their attempt to temporally condition another somatic response, sucking. The second purpose of the present study was, therefore, to investigate the effects on HR and FPA of presenting stimuli in a fixed temporal sequence using the methods of paced respiration and selective attention.

\section{SUBJECTS}

Sixteen female volunteers from introductory psychology courses at Michigan State University received extra course credit for serving as Ss. As they appeared at the laboratory, they were assigned to experimental conditions according to a predetermined random schedule.

\section{APPARATUS}

Stimuli were produced by a modified Crystal Lab Metronoma that presented a neon light at the rate of 32 flashes/min. The neon light from the Metronoma was mounted on a panel that was positioned in front of $S$. The duration of each trial and the ITI were programmed and presented by means of magnetic tape (Porges \& Fitzgerald, in press). Ambient noise level of the experimental room was $40 \mathrm{~dB}$. room temperature was $70^{\circ} \mathrm{F}$. To mask background noise, $60-\mathrm{dB}$ white noise was played through earphones placed on each S. Physiological responses were continuously recorded on a Beckman Type RS Dynograph at a paper speed of $5 \mathrm{~mm} / \mathrm{sec}$. EKG recording sites were cleaned with $70 \%$ ethanol prior to application of the electrodes. HR was measured from EKG Lead II using a Beckman 9857 cardiotachometer. Finger vasomotor activity was measured with a photoelectric plethysmograph. The pickup consisted of a block of black phenolic plastic in which were mounted a Clairex CL 704 photocell and a General Electric 683 miniature lamp powered by a $3 \cdot \mathrm{V}$ battery. The pickup was mounted flush to the tip of the finger by means of a plastic clamp. This arrangement held the surface of the pickup securely against S's fingertip with relatively light, constant pressure, which prevented discomfort to $S$ and occlusion of the local blood vessels. The signal from the plethysmograph bridge was recorded ac.

\section{PROCEDURE}

The Ss were assigned randomly to one of two groups, eight Ss per group, Ss in the paced-respiration group (PR) were instructed to pace their breathing rate at 16 respiration cycles per minute during each trial by inhaling and exhaling in rhythm with the flashing light. Ss in the attentive-observation group (ATT) were instructed to count the number of light flashes during each trial. Each $S$ received 10 trials. The ITI for each experimental group was a constant $20 \mathrm{sec}$. The duration of each trial presentation ranged from 10 to $20 \mathrm{sec}$, with a mean of $15 \mathrm{sec}$.

\section{DATA QUANTIFICATION}

The responses during each of the 10 trials were scored for HR and FPA. Each trial was divided into two periods: a preperiod, consisting of the first 10 beats prior to stimulus onset, and an evoked period, consisting of the first 10 beats following stimulus onset. HR was obtained from the beat-by-beat readout of the cardiotachometer, which transformed the R-R intervals into HR. HR and FPA responses were evaluated by a comparison of means, variances, and beat-by-beat analysis during both periods. FPA was measured beat by beat in terms of millimeters of pen deflection for each pulse and corrected for amplifier gain.

\section{RESULTS}

Heart Rate

Combining the 20 beats of both periods, there was a significant decrease in mean $\mathrm{HR}$ as a function of trials $[\mathrm{F}(9,126)=3.7$, $\mathrm{p}<.005]$. Although the two tasks produced no differential effects on mean HR $(F<1)$, Group PR exhibited mean HR acceleration, while Group ATT exhibited mean HR deceleration to stimulus onset. The reliability of this group difference was supported by a significant Group by Period interaction $[F(1,14)=4.9, p<.045]$. The HR response during the two periods was evaluated by a heat-by-beat analysis. Figure 1 illustrates the mean HR of each beat of both periods as the magnitude of change in beats per minute from the last 
Fig. 1. Mean heart-rate change during the preperiod and evoked period as a function of beats for Group PR and Group ATT.

beat of the preperiod. Although the two groups did not show significant differences in the beat-by-beat patterns, there was a significant difference between the shapes of the HR pattern for the two periods $[F(9,126)=2.2, \quad p<.025]$, indicating significant anticipatory $\mathrm{HR}$ deceleration. Moreover, as illustrated in Fig. 1, HR variability was significantly greater in Group PR $[F(1,14)=5.37, \mathrm{p}<.05]$.

Finger Pulse Amplitude

There was a significant increase in FPA as a function of trials $[F(9,126)=3.1$, $p<.005]$. The tasks produced no differential effects on mean FPA $(F<1)$. However, Group PR exhibited vasoconstriction to stimulus onset, while Group ATT exhibited vasodilation to stimulus onset. Although not statistically significant, this was demonstrated by the direction of the Group by Period interaction $[F(1,14)=3.6, p<.08]$.

\section{DISCUSSION}

Assuming that sequential counting of light flashes required Ss in Group ATT to attend to the external stimulus, and that the task requirements were indeed different for the two groups, the results of the present study support the research cited above by indicating that the direction of the HR response is task dependent, i.e., Ss in the paced respiration group evidenced cardiac acceleration to stimulus onset, while those in the selective-attention group evidenced cardiac deceleration. Although the response to trial onset was task dependent, the anticipatory HR deceleration more specifically appears to be a function of the fixed ITI (or more explicitly, the temporal CS), with the resulting responses reflecting temporal conditioning. This is congruent with the decelerative $H R$ response preceding $C S$ presentation found by Hastings \& Obrist (1967) in their study of interstimulus-interval effects on conditioned heart rate.

There was no evidence for temporally conditioned FPA. Nevertheless, considering the qualitative nature of photoplethysmographically recorded FPA, one cannot conclude that temporal conditioning of FPA is not possible. Since summated data characteristic of between-groups designs tend to mask individual differences, a nonparametric design emphasizing the direction of individual differences may be more appropriate for evaluating this response.

In agreement with physiological evidence, psychophysiological research has shown the finger vasomotor response to be

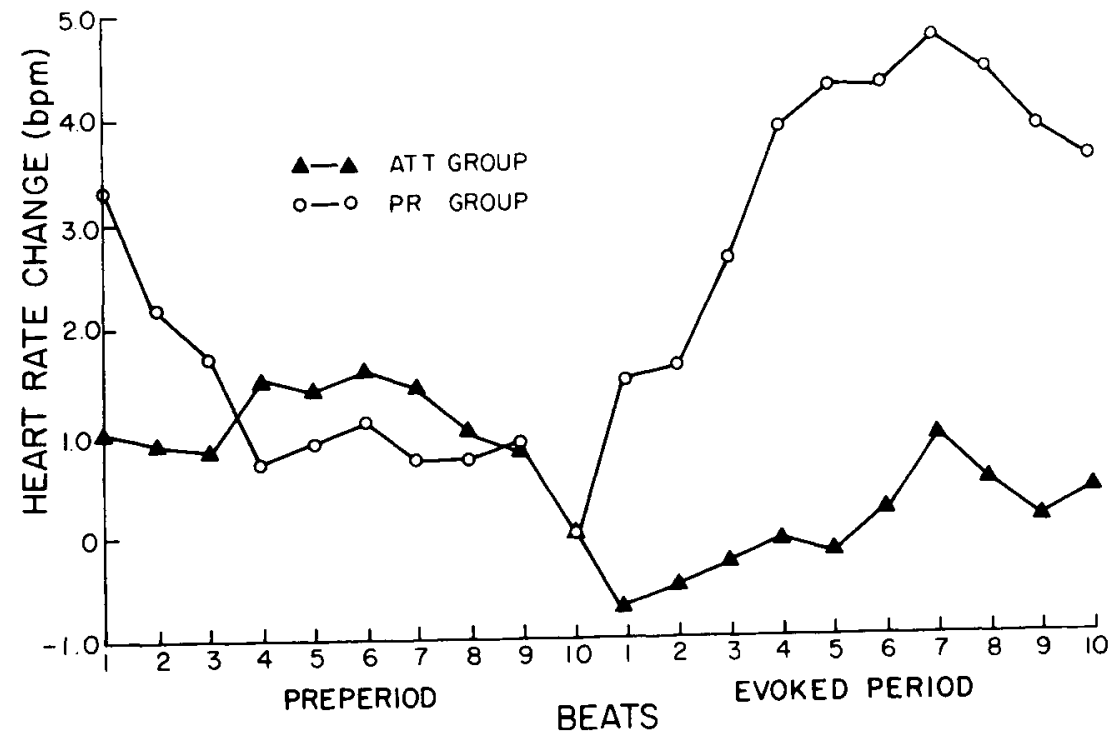

sympathetically innervated nonspecific constriction, i.e., vasomotor control of cutaneous blood flow is solely constrictor (Gaskell, 1956; Roddie, Shepherd, \& Whelan, 1957). Since this peripheral vasomotor constriction is independent of type of stimulation, the increase in FPA following stimulus onset observed in Group ATT might easily be inappropriately interpreted as vasodilation. An alternative interpretation would suggest that for Group ATT, stimulus onset marked the point at which FPA anticipatory constriction terminated. Thus, FPA vasodilation observed at stimulus onset more accurately represents inhibition of preperiod sympathetic anticipatory vasoconstriction, i.e., a release of sympathetic tone.

Group differences in HR variability can be attributed to task requirements, paced respiration increasing sinus arrhythmia and its effect on HR fluctuation. The significant decrease in HR and increases in FPA may be attributed to habituation to the experimental situation and are consistent with previous research (Porges \& Raskin, 1969).

\section{REFERENCES}

ABRAHAMSON, D., BRACKBILL, Y., CARPENTER, R., \& FITZGERALD, H. E. Interaction of stimulus and response in infant conditioning. In H. E. Fitzgerald and $Y$. Brackbill (Eds.), Design and methodology in infant research. Chicago: University of Chicago Press, in press.

BRACKBIL L, Y., LINTZ, L. M., \& FITZGERALD, H. E. Differences in the autonomic and somatic conditioning of infants. Psychosomatic Medicine, 1968, 30, 193-201.

BRENER, J., \& HOTHERSALL, D. Heart rate control under conditions of augmented sensory feedback. Psychophysiology, 1966, 3, 23-28.

FITZGERALD, H. E., LINTZ, L. M., BRACKBILL, Y., \& ADAMS, G. Time perception and conditioning an autonomic response in young human infants. Perceptual $\dot{\&}$ Motor Skills, 1967, 24, 479-486.

GANTT, W. H. Cardiac conddtional reflexes to time. Transactions of the American Neurological Society, 1946, 166.

GASKELL, P. Are there sympathetic vasodilator nerves to the vessels of the hand? Journal of Physiology, 1956, 131, 647-656.

HASTINGS, S. E., \& OBRIST, P. A. Heart rate during conditioning in humans: Effect of varying the interstimulus (CS-UCS) interval. Journal of Experimental Psychology, 1967, 74, 431-442.

HNATIOW, M., \& LANG, P. J. Learned stabilization of cardiac rate. Psychophysiology, $1965,1,330-336$.

LACEY, J. I. Psychophysiological approaches to the evaluation of psychotherapeutic process and outcome. In E. A. Rubinstein and M. B. Parloff (Eds.), Research in psychotherapy. Washington, D. C.: American Psychological Association, 1959, Pp. 160-208. preliminary report of temporal conditioning to three types of neonatal stimulation. Paper presented at the meeting of the Society for Research in Child Development, New York, March 1967.

LOCKHART, R. A. Temporal conditioning of GSR. Journal of Experimental Psychology, 1966, 71, 438-446.

PORGES, S. W., \& FITZGERALD, H. E. An inexpensive method for programming stimuli using magnetic tape. Psychophysiology, in press.

PORGES, S. W., \& RASKIN, D. C. Respiratory and heart rate components of attention. Journal of Experimental Psychology, 1969, 8, 497-503.

RODDIE, I. C., SHEPHERD, J. T., \& WHELAN, R. F. A comparison of the heat elimination from the normal and nerveblocked finger during body heating. Journal of Physiology, $1957,38,445-448$.

NOTE

1. This research was supported in part by a Michigan State University research grant to the first author and by a USPHS predoctoral research fellowship, MH 43834, to the second author. Address requests for reprints to: Hiram $\mathrm{E}$. Fitzgerald, Department of Psychology, Michigan State University, East Lansing, Mich. 48823.
LIPSITT, L. P., \& AMBROSE, J. A. A 